\title{
CHOREOATHETOSIS SECONDARY TO LEAD TOXICITY
}

\author{
Mariana Spitz', Leandro Tavares Lucato², Mônica Santoro Haddad', Egberto Reis Barbosa
}

Firearm projectiles have been described, albeit rarely, as a cause of lead toxicity. The usual route of lead exposure is oral ingestion, but toxicity secondary to retained bullet fragments has been well documented'. Switz et al. described the first case in 1976 - a bullet was lodged in a patient's left ankle and gastrointestinal symptoms emerged forty years after the wound ${ }^{2}$. Since then several reports followed, including one by Goodheart et al. ${ }^{3}$, in 1999, in which the authors reported 25 patients with lead intoxication secondary to intentional petrol sniffing, among whom 8 presented with chorea, though the most common neurological complication was altered mental state, observed in all subjects. The major clinical manifestations of lead toxicity are gastrointestinal, hematological and neurological, namely abdominal cramps, anorexia, nausea, vomiting, constipation, anemia, headache, peripheral neuropathy and encephalopathy ${ }^{4}$. This is characterized by delirium, seizures, somnolence, and even coma. Joint and muscle pain and nephropathy may also be part of the clinical picture ${ }^{5}$.

We herein describe an interesting case, where choreoathetosis was attributed to lead toxicity due to retained bullet fragments.

\section{CASE}

A 42-year-old right-handed man was shot in 1987 at the age of 23. He had been a drug addict since the age of 14 - he had used marijuana and inhaled cocaine, but denied intravenous drug use. He was shot in the left side of the abdomen, left shoulder and right thigh. At that time, he had undergone emergency surgery - there was no injury to internal organs, but one of the bullets could not be successfully removed because its location in the lumbar spinal column - in close proximity to the spinal cord - was considered too risky. The patient was discharged a week after the surgery.

He remained asymptomatic until February 1994, when he started complaining of frequent abdominal cramps, nausea, vomiting and constipation. On that occasion he was referred to the Orthopedics Clinic of our hospital. On physical examination, he was pale, the abdomen was diffusely tender to palpation, but there were no signs of peritonitis. Neurological examination was normal. His blood exams showed normochromic normocytic anemia and blood lead levels were in the toxic range (112 $\mu \mathrm{g} /$ $\mathrm{dL}$ ), which was attributed to the retained bullet. He was diagnosed with lead toxicity and underwent chelation with dimercaprol and EDTA (ethylenediaminetetracetic acid). One month later, since the blood lead levels remained high $(65 \mu \mathrm{g} / \mathrm{dL})$, che-
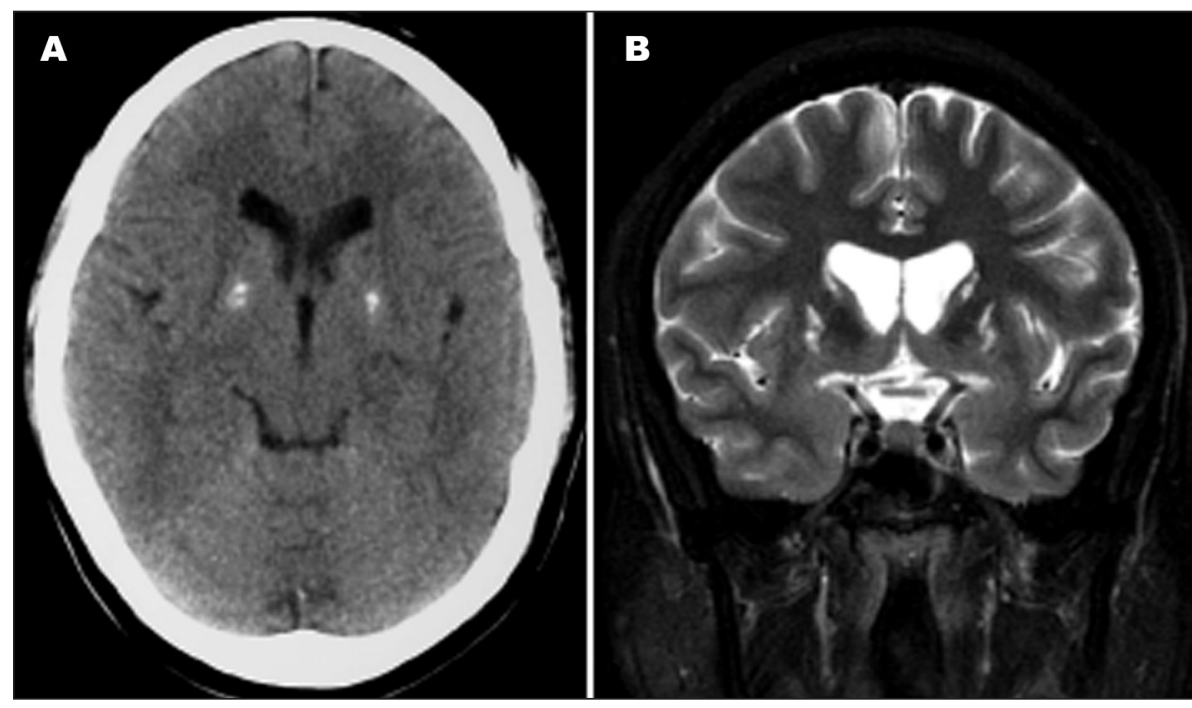

Fig 1. Neuroimaging results. (A) CT demonstrates bilateral and symmetric pallidal calcifications. (B) Coronal T2-weighted image from MR exam depicts volumetric reduction of both putamen and caudate nuclei, presenting hyperintensity in a bilateral and symmetric fashion.

\section{COREOATETOSE SECUNDÁRIA A INTOXICAÇÃO POR CHUMBO}

Departments of Neurology ${ }^{1}$ and Radiology ${ }^{2}$, University of São Paulo Medical School, São Paulo SP, Brazil. Received 29 February 2008, received in final form 8 May 2008. Accepted 30 May 2008.

Dra. Mariana Spitz - Rua Paulo César de Andrade 200/402 - 22221-090 Rio de Janeiro RJ - Brasil. E-mail: marianaspitz@gmail.com 


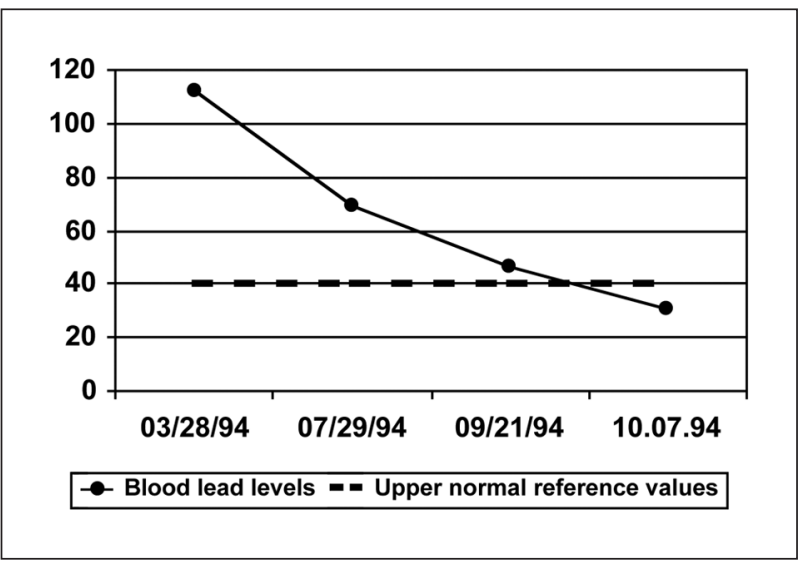

Fig 2. Decrease of patient's blood lead levels ( $\mu \mathrm{g} / \mathrm{dL}$ ) after prescription of chelation therapy.

lation with EDTA was administered again, followed by an unsuccessful attempt to surgically remove the bullet from the spine. There was improvement of the abdominal cramps, but within a few weeks of the procedure, the patient noticed involuntary movements of the hands, trunk and feet. He was then admitted to the Neurologic Clinic for diagnostic evaluation.

The patient is the first child of non-consanguineous parents. His family history is negative for neurological diseases. On neurological examination, there were generalized, partially-suppressible choreoathetoid movements involving the face and proximal limbs, in addition to the toes and fingers. He had a dystonic posture on the right upper limb. His muscle strength, deep tendon reflexes and cognitive function were normal. There was rigidity in the four limbs and plantar reflexes were flexor.

Blood exams were unremarkable, including liver and thyroid function and rheumatologic tests. HIV antibody testing was negative, as well as hepatitis serology. Serum and urinary (24 hour collection) copper and serum cerulopasmin were within normal limits. Huntington disease genetic testing was negative. Electroneuromyography of the four limbs was normal. Cerebrospinal fluid (CSF) analysis revealed normal protein and glucose levels, negative VDRL, no red blood cells and 3 leucocytes. Computed tomography performed in 1996 showed bilateral and symmetric pallidal calcifications and a slight hypoattenuation in both putamen and caudate nuclei. He also underwent brain MRI, which demonstrated some volumetric reduction in both caudate and lentiform nuclei, associated with bilateral and symmetric $\mathrm{T} 2$ hyperintensity and $\mathrm{T} 1$ hypointensity of these structures (Fig 1). Blood lead levels were still increased $(69 \mu \mathrm{g} / \mathrm{dL})$ and another course of EDTA was prescribed.

The patient was given chelation therapy with EDTA for four months and blood lead levels eventually returned to normal (Fig 2). Abnormal movements persisted and were only controlled with neuroleptics. Patient is currently taking haloperidol $7 \mathrm{mg}$ daily. Since the beginning of its administration, the patient tried to withdraw the drug on several occasions, resulting in significant worsening of the choreoathetosis.

\section{DISCUSSION}

Although it is not common in clinical practice, movement disorders can occur following exposure to several chemical substances, such as heavy metals, pesticides and organic solvents. Goldings et al. in 1982 described the case of a 15 year-old boy who inhaled gasoline and developed chorea, myoclonus and ataxia due to lead encephalopathy ${ }^{6}$. Goodheart et al. in 1999 also reported patients with petrol sniffing history, lead toxicity and chorea ${ }^{3}$, but to our knowledge no cases of choreoathetosis secondary to lead toxicity because of a retained bullet have been published to date.

In the case herein described neurological symptoms consisted of choreoathetosis and were ascribed to lead toxicity due to a retained bullet lodged in the spinal column for the past 7 years. But could the patient develop lead toxicity after such a long interval from the gunshot wound? It has been demonstrated that blood lead levels tend to increase with time after injury in patients with projectile retention ${ }^{7}$.Onset of symptoms from the initial gunshot wound has been reported from 2 days up to 52 years ${ }^{1}$.

The pathophysiology of lead absorption from retained bullets is still unclear, but there seems to be a correlation between bullet location and lead toxicity ${ }^{7}$. Synovial and bony interfaces and contact with pleural and CSF have been associated with elevated absorption rates ${ }^{1}$. As the onset of symptoms may be insidious and the asymptomatic interval may be prolonged, a high index of suspicion is required to diagnose lead toxicity ${ }^{8}$.

In 1997 Centers for Disease Control and Prevention defined elevated blood lead levels for children as exceeding $10 \mu \mathrm{g} / \mathrm{dL}^{9}$. In adults, severe lead toxicity is associated with blood lead concentrations of $100 \mu \mathrm{g} / \mathrm{dLl}$ or more ${ }^{10}$. This patient had lead levels of up to $112 \mu \mathrm{g} / \mathrm{dL}$, consistent with severe toxicity.

$\mathrm{CT}$ in this case disclosed pallidal calcifications, bilateral and symmetric, which is a relatively common finding in patients over age $40^{11}$. However, the patient was only 32 years old by the time the first CT was performed, which raises the possibility that these calcifications could be associated to lead intoxication. Calcifications are commonly described in this condition, especially in the chronic form (as is the case described here). They are usually linear in the subcortical area and they can be seen also in the cerebellum and basal ganglia ${ }^{12}$.

MR imaging showed in detail striatal lesions which are probably the substrate for the choreoathetoid movements. There was a clear decrease in volume of the caudate nuclei and also of the putamen, associated to signal abnormalities that are unspecific, but associated to the 
atrophy of these structures, can be related to gliosis and end-stage changes. The irreversibility of the clinical picture corroborates the imaging aspects, although reversibility can sometimes be appreciated in this entity, mainly in acute forms ${ }^{13}$. MR findings in lead toxicity are variable, including white matter, basal ganglia, insula and thalamus involvement ${ }^{13,14}$ - usually presenting T2-hyperintensity. Strictly from an imaging standpoint, differential diagnosis in this case includes diseases with basal ganglia involvement, especially Wilson disease, which was excluded after other diagnostic tests.

We cannot certify that movement disorders and intracranial lesions were due to the lead toxicity, but the temporal course certainly suggests it. There remains a possibility that the neurological symptoms were not the direct effect of the bullet, but we believe that lead poisoning was due to the bullet lodged in the spinal cord and direct penetration into the CSF compartment. We came to the conclusion that the cause of choreoathetosis was lead toxicity considering that other common causes were excluded.

In this case there was no regression of the involuntary movements despite normalization of blood lead levels. After prolonged exposure to lead, effects do not appear to be reversible even if blood lead levels are lowered with chelation ${ }^{15}$.

Lead toxicity is a very rare cause of choreoathetosis. There must be surveillance for people with retained bul- lets concerning the possible development of lead toxicity, since this is a difficult diagnosis.

\section{REFERENCES}

1. Coon T, Miller M, Shirazi F, Sullivan J. Lead toxicity in a 14-year-old female with retained bullet fragments. Pediatrics 2006; 117: 227-230.

2. Switz DM, Elmorshidy ME, Deyerle WM. Bullets, joints, and lead intoxication: a remarkable and instructive case. Arch Intern Med 1976; 136: 939-941.

3. Goodheart RS, Dunne JW. Petrol sniffer's encephalopathy: a study of 25 patients. Med J Aust 1994; 160: 178-181.

4. Brodkin E, Copes R, Mattman A, Kennedy J, Kling R, Yassi A. Lead and mercury exposures: interpretation and action. CMAJ 2007; 176: 59-63.

5. Ford MD. Clinical toxicology. Philadelphia: Saunders, 2001.

6. Goldings AS, Stewart RM. Organic lead encephalopathy: behavioral change and movement disorder following gasoline inhalation. J Clin Psychiatry 1982; 43: 70-72.

7. Ibrahim D, Froberg B, Wolf A, Rusyniak DE. Heavy metal poisoning clinical presentations and pathophysiology. Clin Lab Med 2006; 26: 6797, viii.

8. McQuirter JL, Rothenberg SJ, Dinkins GA, Manalo M, Kondrashov V, Todd AC. The effects of retained lead bullets on body lead burden. J Trauma 2001; 50: 892-899.

9. Centers for Disease Control and Prevention. Preventing lead poisoning in young children. Atlanta; CDC, 2005.

10. Gracia RC, Snodgrass WR. Lead toxicity and chelation therapy. Am J Health Syst Pharm 2007; 64: 45-53.

11. Teo JG, Goh KY, Ahuja A, et al. Intracranial vascular calcifications, glioblastoma multiforme, and lead poisoning. AJNR Am J Neuroradiol 1997; 18: 576-579.

12. Reyes PF, Gonzalez CF, Zalewska MK, Besarab A. Intracranial calcification in adults with chronic lead exposure. AJR Am J Roentgenol 1986; 146: 267-270.

13. Atre AL, Shinde PR, Shinde SN, et al. Pre- and posttreatment MR imaging findings in lead encephalopathy. AJNR Am J Neuroradiol 2006; 27: 902-903.

14. Tuzun M, Tuzun D, Salan A, Hekimoglu B. Lead encephalopathy: CT and MR findings. J Comput Assist Tomogr 2002; 26: 479-481.

15. Carpenter DO. Effects of metals on the nervous system of humans and animals. Int J Occup Med Environ Health 2001; 14: 209-218. 